\title{
Targeted activation of PKA and Epac promotes glioblastoma regression in vitro
}

\author{
NAOTOSHI SUGIMOTO ${ }^{1}$, SHINJI MIWA ${ }^{2}$, HIROYUKI TSUCHIYA ${ }^{2}$, YOSHIAKI HITOMI ${ }^{3}$, \\ HIROYUKI NAKAMURA ${ }^{3}$, AKIHIRO YACHIE ${ }^{4}$ and SHOICHI KOIZUMI ${ }^{5}$
}

\author{
Departments of ${ }^{1}$ Physiology, ${ }^{2}$ Orthopedic Surgery, ${ }^{3}$ Public Health and ${ }^{4}$ Pediatrics, Graduate School of Medical Science; \\ ${ }^{5}$ United Graduate School of Child Development, Kanazawa University, Kanazawa, Ishikawa 920-8640, Japan
}

Received July 12, 2012; Accepted January 8, 2013

DOI: $10.3892 / \mathrm{mco} .2013 .65$

\begin{abstract}
Ras-p44/42 mitogen-activated protein kinase (MAPK) and Akt signaling are the key pathways involved in the promotion of glioblastoma formation. Notably, phosphodiesterase 4 (PDE4) is widely expressed in brain tumors and promotes their growth. PDE4 inhibitors have been reported to suppress glioblastoma growth in vitro and in vivo. The mechanisms underlying these actions, however, have yet to be elucidated. The aim of this study was to investigate whether intracellular cyclic adenosine monophosphate (cAMP) was able to suppress the Ras-p44/42 MAPK signaling pathway via protein kinase A (PKA) and exchange protein directly activated by cAMP (Epac) in U87MG human malignant glioma cells. Forskolin, an activator of adenylate cyclase, inhibited cell growth and the phosphorylation of p44/42 MAPK in U87MG cells, whereas the non-hydrolyzable cAMP analog 8-bromoadenosine 3',5'-cAMP (8-Br-cAMP) considerably suppressed cell growth and phosphorylation of p44/42 MAPK. The inhibitory effects of forskolin were partially prevented by the PKA inhibitor H89. The Epac-selective agonist 8-(4-chlorophenylthio)-2'- $O$-methyladenosine cAMP (8-CPT-cAMP) inhibited phosphorylation of p44/42 MAPK. These findings suggest that PKA and Epac are involved in the effect of intracellular cAMP on the Ras-p44/42 MAPK signaling pathway.
\end{abstract}

\section{Introduction}

Ras and Akt are signaling proteins that mediate fundamental aspects of normal growth and development in various organs. However, when the Ras and Akt pathways become extremely

Correspondence to: $\mathrm{Dr}$ Naotoshi Sugimoto, Department of Physiology, Graduate School of Medical Science, Kanazawa University, 13-1 Takara-machi, Kanazawa, Ishikawa 920-8640, Japan

E-mail: ns@med.kanazawa-u.ac.jp

Key words: cyclic adenosine monophosphate, protein kinase A, exchange protein activated by cyclic adenosine monophosphate, glioblastoma, mitogen-activated protein kinase active, malignant transformation of normal tissue may occur (1-3). For instance, Holland et al (4) demonstrated that the combined activation of Ras and Akt induces glioblastoma formation in mice. Therefore, Ras and Akt signaling pathways have emerged as attractive targets for the treatment of numerious types of tumors including glioblastoma $(3,5,6)$.

Glioblastoma is the most common type of brain tumor, it is highly malignant and exhibits aggressive invasive growth. Common genetic abnormalities, including Ras and phosphatase and tensin homolog (PTEN) deleted on chromosome 10, in glioblastoma, are associated with the aberrant activation or suppression of cell signal transduction pathways and resistance to radiation and chemotherapy $(2,3)$. Notably, phosphodiesterase 4 (PDE4) is widely expressed in brain tumors and promotes their growth (7). Brain region-specific differences in cyclic adenosine monophosphate (cAMP) levels have been reported to account for the pattern of gliomagenesis, while low levels of cAMP promote glioma formation in neurofibromatosis-1 genetically engineered mouse models $(8,9)$. PDE4 inhibitor has been shown to suppress glioblastoma growth in vitro and in xenograft models $(7,10,11)$.

cAMP is considered to be a ubiquitous regulator of inflammatory and immunological reactions (12-14), modulating several physiological processes via the activation of protein kinase A (PKA) and the exchange protein directly activated by cAMP (Epac). PKA and Epac are molecular players that are downstream of cAMP $(14,15)$. PDE inhibitors have been reported to increase cellular cAMP levels and, in turn, prevent Akt activity in rat glial and human osteosarcoma cells $(15,16)$.

In the present study, the effects of cAMP on Ras and Akt signaling pathways were examined in U87MG human malignant glioma cells. cAMP was demonstrated to suppress cell growth and p44/42 mitogen-activated protein kinase (MAPK) activity, which is downstream of the Ras signaling pathway, but not Akt activity in a PKA- and Epac-dependent manner. Findings of this study provide a mechanistic basis for developing therapeutic approaches for treating or preventing brain tumors.

\section{Materials and methods}

Chemicals. Forskolin and Dulbecco's modified Eagle's medium (DMEM) were obtained from Wako Pure Chemical 
A

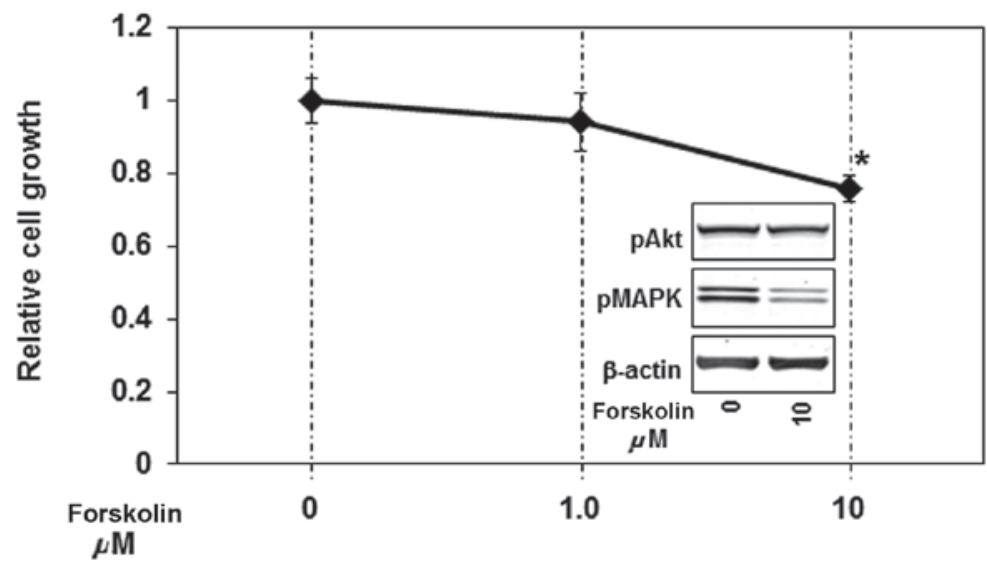

B
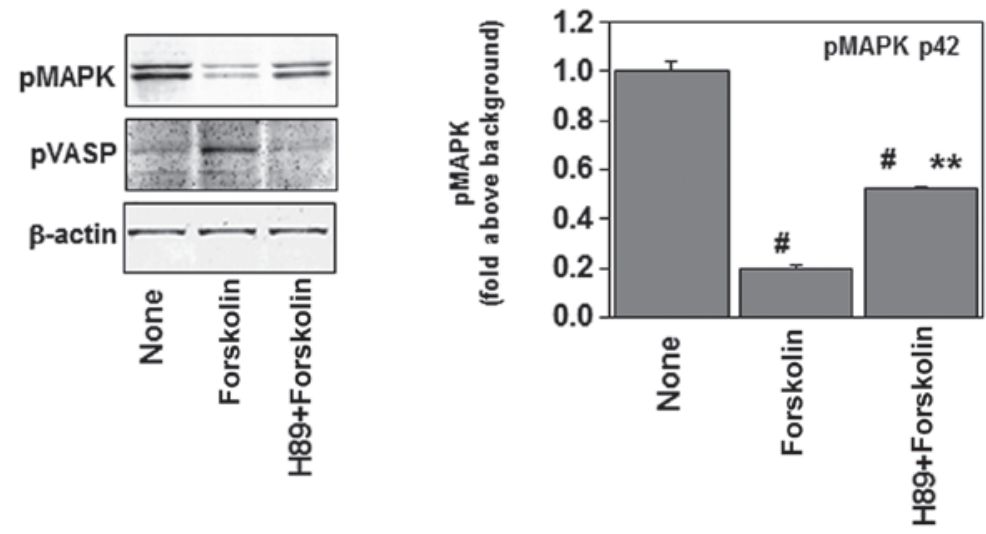

Figure 1. Change in cell growth and the level of phospho-Akt and phospho-p44/42 mitogen-activated protein kinase (MAPK) (A) in U87MG human malignant glioma cells after treatment with Forskolin (1-10 $\mu \mathrm{M})$ is shown. Change in the level of phospho-p44/42 MAPK and phospho-vasodilator-stimulated phosphoprotein (VASP) after treatment with Forskolin $(10 \mu \mathrm{M})$, a combination of Forskolin $(10 \mu \mathrm{M})$ and $\mathrm{H} 89(10 \mu \mathrm{M})$, or H89 $(10 \mu \mathrm{M})(\mathrm{B})$ in U87MG cells is shown. Each column represents the mean \pm standard error of the mean $(\mathrm{SEM}) .{ }^{*} \mathrm{P}<0.05,{ }^{\sharp} \mathrm{P}<0.01$ vs. untreated control, ${ }^{* *} \mathrm{P}<0.05$ vs. Forskolin treatment.

Industries, Ltd. (Osaka, Japan). 8-Bromoadenosine 3',5'-cAMP (8-Br-cAMP) and 8-(4-chlorophenylthio)-2'- $O$-methyladenosine cAMP (8-CPT-cAMP, Epac activator) were obtained from Biaffin GmbH \& Co. KG (Kassel, Germany). Anti-phospho-specific vasodilator-stimulated phosphoprotein (VASP) (Ser157) and H89 were obtained from Calbiochem (La Jolla, CA, USA). Fetal bovine serum (FBS) was obtained from Invitrogen Corporation (Carlsbad, CA, USA). Anti-phospho-specific Akt (Ser473), anti-phospho-specific p44/42 MAPK (Thr202/Tyr204), anti- $\beta$-actin, horseradish peroxidase (HRP)-linked anti-rabbit IgG, and anti-mouse IgG were purchased from Cell Signaling Technology, Inc. (Danvers, MA, USA).

Cell culture. U87MG human glioblastoma cells were provided by Dr Nakata (Kanazawa University, Kanazawa, Japan). Cells were maintained in DMEM containing $10 \% \mathrm{FBS}$ at $37^{\circ} \mathrm{C}$ in a $5 \% \mathrm{CO}_{2}$ incubator.

Western blot analysis. Western blot analysis was performed as described previously $(14,17)$.

Cell proliferation assay. Cell proliferation was analyzed using the Cell Counting kit 8 (Wako Pure Chemical Industries, Ltd., Tokyo, Japan). U87MG human malignant glioma cells were seeded in 96-well plates at a density of $1 \times 10^{3}$ cells/ well. Following a 24-h incubation, the cells were treated with forskolin or 8-Br-cAMP for $24 \mathrm{~h}$. The cells were then incubated with $10 \mu \mathrm{l}$ WST- 8 for $2 \mathrm{~h}$. Absorbance of the colored formazan product produced by mitochondrial dehydrogenases in metabolically active cells was recorded at $450 \mathrm{~nm}$ as the background value. Cell proliferation was expressed as a percentage of absorbance obtained in treated wells compared with untreated (control) wells.

Statistical analysis. Data were presented as the means \pm standard error of the mean (SEM) from at least three independent experiments. Statistical analysis was performed by analysis of variance (ANOVA), followed by Dunnett's test. $\mathrm{P}<0.05$ or $\mathrm{P}<0.01$ were considered to indicate a statistically significant difference.

\section{Results}

Forskolin downregulates cell growth. Initially, we examined the effects of forskolin, an activator of adenylate cyclase, on cell growth. As shown in Fig. 1A, $10 \mu \mathrm{M}$ of forskolin, but not $1 \mu \mathrm{M}$, slightly but markedly inhibited cell growth $(\sim 20 \%)$ in U87MG human glioblastoma cells. Concomitantly, forskolin increased the expression of phospho-vasodilator-stimulated phosphoprotein (phospho-VASP), indicating an elevation of intracellular cAMP levels (Fig. 1B) $(18,19)$. 
A
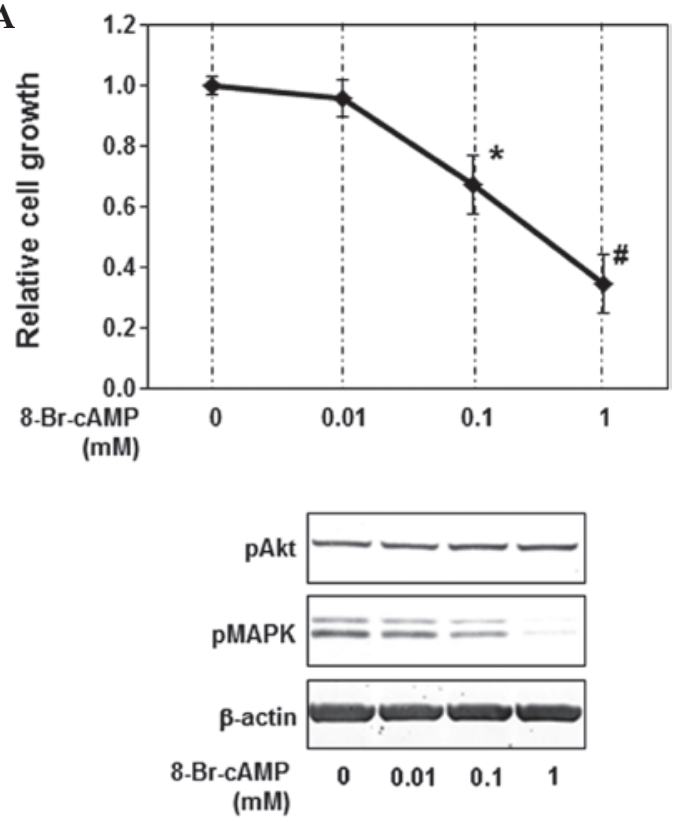

B
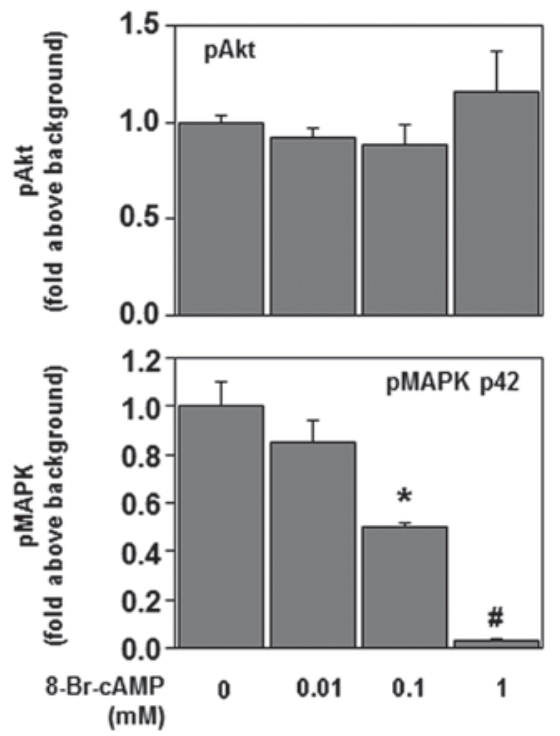

Figure 2. Change in cell growth (A) and the level of phospho-Akt and phospho-p44/42 mitogen-activated protein kinase (MAPK) (B) following treatment with 8-bromoadenosine $3^{\prime}, 5^{\prime}$-cAMP (8-Br-cAMP) $(0.01-1 \mathrm{mM})$ in U87MG cells. Each column shows the mean \pm standard error of the mean (SEM). ${ }^{*} \mathrm{P}<0.05,{ }^{, "} \mathrm{P}<0.01$ vs. untreated controls.

Forskolin inhibits p44/42 MAPK but not Akt activity. Ras signals to Raf, mitogen-induced extracellular kinase (MEK), and MAPKs, especially p44/42 MAPK. The effects of forskolin on p44/42 MAPK and Akt were examined. Treatment with $10 \mu \mathrm{M}$ of forskolin inhibited phosphorylation of p44/42 MAPK but not Akt in U87MG (Fig. 1A, inset figure), indicating that the Ras-p44/42 MAPK signaling pathway is inhibited by forskolin. PKA and Epac are molecular players downstream of cAMP. As shown in Fig. 1B, the effects of the PKA inhibition completely by $\mathrm{H} 89$ blocked the forskolin-induced phosphorylation of VASP, while partially preventing the forskolin-induced inhibition of phosphorylation of p44/42 MAPK. These results indicate that PKA is partially involved in the effects of forskolin on the Ras-p44/42 MAPK signaling pathway.
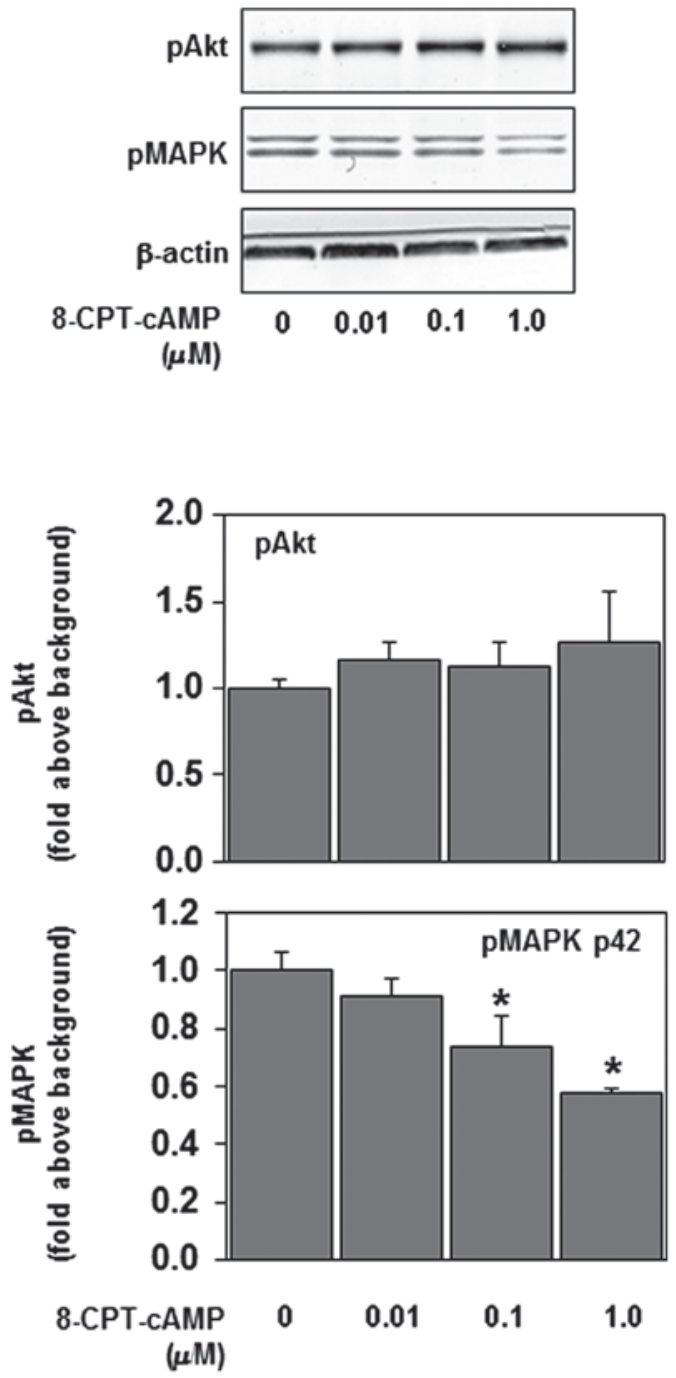

Figure 3. Change in the level of phospho-Akt and phospho-p44/42 mitogen-activated protein kinase (MAPK), following treatment with 8-(4-chlorophenylthio)-2'- $O$-methyladenosine cAMP (8-CPT-cAMP) $(0.01-1 \mu \mathrm{M})$ in U87MG cells. Each column shows the mean \pm standard error of the mean (SEM). ${ }^{*} \mathrm{P}<0.05$ vs. untreated controls.

Cell-penetrating cAMP analog inhibits cell growth and p44/42 MAPK, but not Akt activity. To determine whether intracellular cAMP is involved in the inhibitory effect of forskolin, we examined the effects of the non-hydrolyzable cAMP analog 8-Br-cAMP on cell growth and phosphorylation of p44/42 MAPK and Akt. In U87MG, in which PDE4 expression is upregulated (20), the non-hydrolyzable cAMP analog 8-Br-cAMP might be more effective compared with forskolin stimulation. Therefore, $1 \mathrm{mM}$ of 8-Br-cAMP considerably suppressed cell growth and phosphorylation of p44/42 MAPK (Fig. 2A and B). As shown in Fig. 2, 8-Br-cAMP inhibited cell growth and phosphorylation of p44/42 MAPK in a dose-dependent manner, mimicking the forskolin effect. However, 8-Br-cAMP did not affect the phosphorylation of Akt (Fig. 2B). These results indicate that intracellular cAMP is involved in the effects of forskolin on cell growth and the Ras-p44/42 MAPK signaling pathway.

cAMP-induced p44/42 MAPK deactivation is Epac-dependent. To examine the potential signaling 
role of Epac, the ability of the Epac-selective agonist 8-CPT-cAMP to inhibit phosphorylation of p44/42 MAPK was examined. 8-CPT-cAMP is a non-hydrolyzable cAMP analog that activates Epac with greater potency compared with PKA (21). Recent studies have shown that concentrations of 8-CPT-cAMP as low as $5 \mu \mathrm{M}$ effectively activate Epac in studies using cultured cells $(14,15)$. In our experiments, 8-CPT-cAMP inhibited the phosphorylation of p44/42 MAPK, but not that of Akt, in a dose-dependent manner (Fig. 3). These results suggest that Epac is involved in the effect of intracellular cAMP on the Ras-p44/42 MAPK signaling pathway.

\section{Discussion}

Ras-p44/42 MAPK signaling is a crucial signaling pathway in the regulation of growth and development in normal tissues and tumors (1-3). Increased activity of Ras-p44/42 MAPK is found in several human glioblastoma $(4,22)$. In the present study, the upregulation of intracellular cAMP has been demonstrated to inhibit p44/42 MAPK, but not Akt activity, and proliferation in human glioblastoma cells in vitro.

Elevated levels of cAMP in the cell lead to the activation of different cAMP targets, including PKA and Epac. The two families of cAMP effectors provide a mechanism for the precise and integrated control of cAMP signaling pathways in a spatial and temporal manner. PKA and Epac may act independently, converge synergistically or oppose each other in regulating a specific cell function $(18-21,23,24)$. In this study, PKA and Epac have been demonstrated to be responsible for cAMP-dependent p44/42 MAPK dephosphorylation (Figs. 1 and 3), confirming that cAMP prevents cell growth through PKA and Epac activation in U172 and U87MG human glioblastoma cells (20).

In a recent study, cAMP was shown to inhibit the activity of Akt through Epac-PTEN pathway activation in glial and osteosarcoma cells $(15,16)$. In this study, however, forskolin or cAMP analogs failed to inhibit Akt activation in U87MG glioblastoma cells (Figs. 1-3). One of the numerous functions of PTEN is the regulation of Akt activity, thus, loss of PTEN function induces Akt hyperactivation (3). Since PTEN is not expressed in U87MG human glioblastoma cells (15), cAMP is likely to fail to inhibit Akt activation in PTEN-depleted U87MG glioblastoma cells.

In their study, Holland et al (4) demonstrated that although activated Ras or Akt alone is insufficient to induce glioblastoma formation, their combination induces glioblastoma formation in mice. Therefore, Ras and Akt signaling pathways have emerged as attractive targets for the treatment of glioblastoma (2-5). PKA and Epac activators as well as PDE4 inhibitors seem to be extremely effective for the treatment of human glioblastoma.

In conclusion, we have shown that cAMP inhibits p44/42 MAPK activity and proliferation in PTEN-depleted human glioblastoma cells in vitro through PKA and Epac activation. However, details regarding the mechanism underlying the PKA and Epac suppression of p44/42 MAPK have yet to be elucidated. Further studies are required to determine the mechanism of PKA and Epac activation-dependent MAPK inhibition in glioblastoma cells.

\section{Acknowledgements}

This study was funded in part by Grants-in-Aid for Science and Culture from the Ministry of Education, Culture, Sports, Science and Technology of Japan.

\section{References}

1. Parsa AT and Holland EC: Cooperative translational control of gene expression by Ras and Akt in cancer. Trends Mol Med 10: 607-613, 2004.

2. McCubrey JA, Steelman LS, Abrams SL, Lee JT, Chang F, Bertrand FE, Navolanic PM, Terrian DM, Franklin RA, D'Assoro AB, Salisbury JL, Mazzarino MC, Stivala F and Libra M: Roles of the RAF/MEK/ERK and PI3K/PTEN/AKT pathways in malignant transformation and drug resistance. Adv Enzyme Regul 46: 249-279, 2006.

3. Hers I, Vincent EE and Tavaré JM: Akt signalling in health and disease. Cell Signal 23: 1515-1527, 2011.

4. Holland EC, Celestino J, Dai C, Schaefer L, Sawaya RE and Fuller GN: Combined activation of Ras and Akt in neural progenitors induces glioblastoma formation in mice. Nat Genet 25: 55-57, 2000.

5. Höland K, Salm F and Arcaro A: The phosphoinositide 3-kinase signaling pathway as a therapeutic target in grade IV brain tumors. Curr Cancer Drug Targets 11: 894-918, 2011.

6. McCubrey JA, Steelman LS, Abrams SL, Bertrand FE, Ludwig DE, Bäsecke J, Libra M, Stivala F, Milella M, Tafuri A, Lunghi P, Bonati A and Martelli AM: Targeting survival cascades induced by activation of Ras/Raf/MEK/ERK, PI3K/PTEN/Akt/mTOR and Jak/STAT pathways for effective leukemia therapy. Leukemia 22: 708-722, 2008.

7. Goldhoff P, Warrington NM, Limbrick DD Jr, Hope A, Woerner BM, Jackson E, Perry A, Piwnica-Worms D and Rubin JB: Targeted inhibition of cyclic AMP phosphodiesterase-4 promotes brain tumor regression. Clin Cancer Res 14: 7717-7725, 2008.

8. Brown JA, Gianino SM and Gutmann DH: Defective cAMP generation underlies the sensitivity of CNS neurons to neurofibromatosis-1 heterozygosity. J Neurosci 30: 5579-5589, 2010.

9. Warrington NM, Gianino SM, Jackson E, Goldhoff P, Garbow JR, Piwnica-Worms D, Gutmann DH and Rubin JB: Cyclic AMP suppression is sufficient to induce gliomagenesis in a mouse model of neurofibromatosis-1. Cancer Res 70: 5717-5727, 2010

10. Yang L, Jackson E, Woerner BM, Perry A,Piwnica-Worms D and Rubin JB: Blocking CXCR4-mediated cyclic AMP suppression inhibits brain tumor growth in vivo. Cancer Res 67: 651-658, 2007.

11. Sengupta R, Sun T, Warrington NM and Rubin JB: Treating brain tumors with PDE4 inhibitors. Trends Pharmacol Sci 32: 337-344, 2011.

12. Moore AR and Willoughby DA: The role of cAMP regulation in controlling inflammation. Clin Exp Immunol 101: 387-389, 1995.

13. Moon EY, Lee JH, Lee JW, Song JH and Pyo S: ROS/Epac1-mediated Rap1/NF-kappaB activation is required for the expression of BAFF in Raw264.7 murine macrophages. Cell Signal 23: 1479-1488, 2011.

14. Saito T, Sugimoto N, Ohta K, Shimizu T, Ohtani K, Nakayama Y, Nakamura T, Hitomi Y, Nakamura H, Koizumi S and Yachie A: Phosphodiesterase inhibitors suppress Lactobacillus casei cell-wall-induced NF- $\mathrm{B}$ and MAPK activations and cell proliferation through protein kinase $\mathrm{A}$ - or exchange protein activated by cAMP-dependent signal pathway. Sci World J 2012: 748572, 2012.

15. Sugimoto N, Miwa S, Ohno-Shosaku T, Tsuchiya H, Hitomi Y, Nakamura H, Tomita K, Yachie A and Koizumi S: Activation of tumor suppressor protein PTEN and induction of apoptosis are involved in cAMP-mediated inhibition of cell number in B92 glial cells. Neurosci Lett 497: 55-59, 2011.

16. Miwa S, Sugimoto N, Shirai T, Hayashi K, Nishida H, Ohnari I, Takeuchi A, Yachie A and Tsuchiya H: Caffeine activates tumor suppressor PTEN in sarcoma cells. Int J Oncol 39: 465-472, 2011. 
17. Sugimoto N, Shido O, Matsuzaki K, Ohno-Shosaku T, Hitomi Y, Tanaka M, Sawaki T, Fujita Y, Kawanami T, Masaki Y, Okazaki T, Nakamura H, Koizumi S, Yachie A and Umehara H: Cellular heat acclimation regulates cell growth, cell morphology, mitogen-activated protein kinase activation, and expression of aquaporins in mouse fibroblast cells. Cell Physiol Biochem 30: 450-457, 2012.

18. Comerford KM, Lawrence DW, Synnestvedt K, Levi BP and Colgan SP: Role of vasodilator-stimulated phosphoprotein in PKA-induced changes in endothelial junctional permeability. FASEB J 16: 583-585, 2002.

19. Loza MJ, Foster S, Peters SP and Penn RB: Beta-agonists modulate T-cell functions via direct actions on type 1 and type 2 cells. Blood 107: 2052-2060, 2006.

20. Moon EY, Lee GH, Lee MS, Kim HM and Lee JW: Phosphodiesterase inhibitors control A172 human glioblastoma cell death through cAMP-mediated activation of protein kinase A and Epac1/Rap1 pathways. Life Sci 90: 373-380, 2012.
21. Enserink JM, Christensen AE, de Rooij J, van Triest M, Schwede F, Genieser HG, Døskeland SO, Blank JL and Bos JL: A novel Epac-specific cAMP analogue demonstrates independent regulation of Rap1 and ERK. Nat Cell Biol 4: 901-906, 2002.

22. Alloussi SH, Alkassar M, Urbschat S, Graf N and Gärtner B: All reovirus subtypes show oncolytic potential in primary cells of human high-grade glioma. Oncol Rep 26: 645-649, 2011.

23. Tamma G, Lasorsa D, Ranieri M, Mastrofrancesco L, Valenti G and Svelto M: Integrin signaling modulates AQP2 trafficking via Arg-Gly-Asp (RGD) motif. Cell Physiol Biochem 27: 739-748, 2011.

24. Gloerich M and Bos JL: Epac: defining a new mechanism for cAMP action. Annu Rev Pharmacol Toxicol 50: 355-375, 2010. 